\title{
TRANSIENT RESPONSE OF SLOSHING FLUID IN A THREE DIMENSIONAL TANK
}

Chih-Hua Wu

Department of Marine Environment and Engineering, National Sun Yatsen University, Kaohsiung, Taiwan, R.O.C

Bang-Fuh Chen

Department of Marine Environment and Engineering, National Sun Yatsen University, Kaohsiung, Taiwan, R.O.C., chenbf@mail.nsysu.edu.tw

Follow this and additional works at: https://jmstt.ntou.edu.tw/journal

Part of the Mechanical Engineering Commons

\section{Recommended Citation}

Wu, Chih-Hua and Chen, Bang-Fuh (2012) "TRANSIENT RESPONSE OF SLOSHING FLUID IN A THREE DIMENSIONAL TANK," Journal of Marine Science and Technology. Vol. 20: Iss. 1, Article 4.

DOI: 10.51400/2709-6998.2419

Available at: https://jmstt.ntou.edu.tw/journal/vol20/iss1/4

This Research Article is brought to you for free and open access by Journal of Marine Science and Technology. It has been accepted for inclusion in Journal of Marine Science and Technology by an authorized editor of Journal of Marine Science and Technology. 


\title{
TRANSIENT RESPONSE OF SLOSHING FLUID IN A THREE DIMENSIONAL TANK
}

\author{
Chih-Hua Wu and Bang-Fuh Chen
}

Key words: 3D tank, liquid sloshing, nonlinear waves, finite difference method.

\begin{abstract}
Sloshing waves in moving tanks have been studied numerically, theoretically and experimentally in the past several decades. Most reported studies have been for tanks excited by forcing motion in a limited number of directions and with fixed excitation frequencies throughout the forcing. In the present study, a time-independent finite difference method is used to simulate fluid sloshing in the three-dimensional tanks with arbitrary depths and the tanks are subject to a range of excitation frequencies with motions that exhibit multiple degrees of freedom.

The developed numerical scheme is verified by rigorous benchmark tests, and the advantage and efficiency of the method is also discussed. The wave motions that arise for a variety of water depths and a range of excitation frequencies are presented and discussed. The coupled motions of surge and sway are simulated with various excitation angles and frequencies. The 'diagonal', 'single-directional', 'square-like', 'swirling' and 'chaotic' waves are successfully obtained in this study and the transient response of sloshing waves in the tank is discussed in detail.
\end{abstract}

\section{INTRODUCTION}

Free surface sloshing in a moving container is associated with various engineering problems, such as tankers on highways, liquid oscillations in large storage tanks caused by earthquakes, sloshing of liquid cargo in ocean-going vessels, and the motion of liquid fuel in aircraft and spacecraft. It is known that partially filled tanks are prone to violent sloshing under certain conditions, especially when near resonant excitation occurs. Excitation with frequencies in the vicinity of the lowest natural frequencies of the liquid motion is of primary practical interest.

Sloshing waves in moving tanks have been studied nu-

Paper submitted 01/19/10; revised 06/18/10; accepted 07/08/10. Author for correspondence: Bang-Fuh Chen (e-mail: chenbf@mail.nsysu.edu.tw).

Department of Marine Environment and Engineering, National Sun Yatsen University, Kaohsiung, Taiwan, R.O.C. merically, analytically and experimentally in the past several decades, and these studies have explored a range of significant phenomena such as linear and nonlinear effects of the sloshing wave and the effect of fluid viscosity. Abramson [1] provides a comprehensive review and discussion of early analytic and experimental studies of liquid sloshing, with application to the aerospace industry. Neglecting fluid viscosity, a potential formulation of the problem has been employed by Nakayama and Washizu [21], Ockendon and Waterhouse [23] and Faltinsen [7] amongst others.

Besides the potential flow approaches, many numerical studies of the problem with primitive variables have been made, particularly when the fully nonlinear effects of the waves on the free surface are included. Papers that describe the modeling of two-dimensional sloshing include Chen and Chiang [5], Aliabadi, Johnson \& Abedi [2], Frandsen [13], and Chen and Nokes [6]. Detailed surveys have been given by Ibrahim et al. [18], who provide general insight into sloshing problems, and by Cariou and Casella [3] who review commercial codes. The advantages and disadvantages of various computational fluid dynamics (CFD) methods are compared in the ISSC Report [18], and Ibrahim et al. [18]. Hit and Nichols [14] developed a method known as the volume of fluid (VOF) method which allows a steep, and highly contorted, free surface. Celebi and Akyuldiz [4] used the VOF technique to simulate which was forced to move harmonically along a vertical curve with rolling motion. Turnbull et al. [24] used simple $\sigma$-transformed mappings in pseudo-spectral and finite element schemes to solve for the free surface motion of fluid in a tank near the resonant frequency. Kim [20] used the finite difference method with SOLA scheme to simulate fluid sloshing in a two and three dimensional liquid containers. The free surface profile was assumed to be a single-valued function (SURF scheme) and the free-slip condition was applied in his computations.

Frandsen [13] developed a fully nonlinear finite difference model based on the inviscid flow equations. He described the sloshing motion in a 2-D wave tank based on potential theory according to a modified $\sigma$-transformation that stretches the grid from the bed to the surface. The advantage of the $\sigma$-transformation is that it can avoid re-meshing due to the moving free surface, and the mapping avoids the necessity to calculate the free surface velocity components explicitly. Moreover, free surface smoothing by means of a spatial filter 
is also not required. More recently, Chen and Nokes [6] studied detailed transient sloshing phenomenon, streamline patterns and excitation frequency effects on hydrodynamic force coefficients.

The analysis of $3 \mathrm{D}$ tanks is relatively rare in the literature. For 3D tank sloshing, Feng [12] used a three-dimensional marker and cell method to study fluid sloshing in a rectangular tank. This method took large amounts of computer memory and CPU time, and the results reported indicate the presence of numerical instabilities. Wu, Ma and Taylor [27] used an inviscid finite element method to analyze fully nonlinear waves in a three dimensional tank.

The aforementioned studies were in tanks excited by a limited range of excitation directions, and with a fixed excitation frequency throughout. In reality, for a liquid tank sitting on the ground when an earthquake occurs, or for a tank floating on the sea, the excitation direction can include multiple degrees of freedom, including surge, sway, heave, pitch, roll and yaw (see Fig. 1).

In the present study, we consider the excitation of a three dimensional tank with different dimensionless excitation amplitudes; with multiple degrees of freedom for the excitation direction; with excitation frequencies near and far from the natural frequency. The main focus of this paper is the response of fluid in a 3-D tank undergoing different combinations of motion with varying excitation directions.

Section 2 introduces the equations of motion which are written in a moving frame of reference attached to the accelerating tank. The fully non-linear free surface boundary conditions are listed in this section. The coordinate transformation functions that map the time-dependent domain into a fixed unit cubic, and allow for mesh stretching at the boundaries, are also presented in this section along with a sensible non-dimensionalization of the governing equations. The proposed finite-difference method is developed in Section 3, where the full iterative procedure is introduced. Section 4 presents the detailed results and provides a comprehensive discussion of all phenomena found in this study. Although the governing equations incorporate excitations with six degrees of freedom, only surge and sway motions are included in the simulations presented here. Section 5 summarizes the key conclusions.

\section{MATHEMATICAL FORMULATION}

The coordinate system is chosen to move with the tank (including surge, sway, heave, yaw, roll and pitch motions) and is illustrated in Fig. 1(a). The inviscid momentum equations can be written as

$$
\begin{aligned}
& \frac{\partial u}{\partial t}+u \frac{\partial u}{\partial x}+v \frac{\partial u}{\partial y}+w \frac{\partial u}{\partial z}=-g_{x}-\frac{1}{\rho} \frac{\partial p}{\partial x}-\ddot{x}_{C}+ \\
& -(\ddot{\beta} z-\ddot{\gamma} y)-\left(2 \dot{\alpha} \dot{\beta}-\dot{\beta}^{2}-\dot{\gamma}^{2}\right) x-2(\dot{\beta} w-\dot{\gamma} v)-\left(\dot{\beta} w_{t}-\dot{\gamma} v_{t}\right)
\end{aligned}
$$

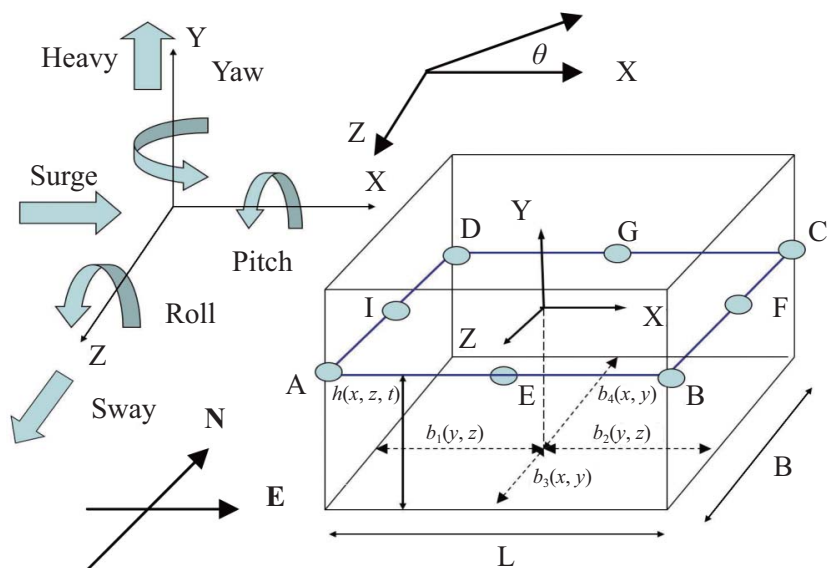

(a)

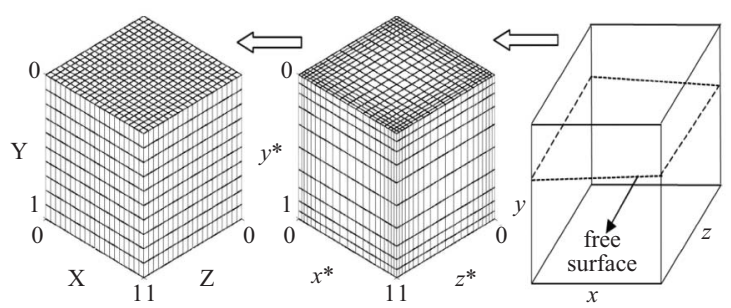

(b)

Fig. 1. (a) Definition of sketch. (b) The concepts of transformation coordinate systems and stretch of the grid. The stretching constants presented in $\left(x^{*}, y^{*}, z^{*}\right)$ coordinate are $\lambda_{1}=\lambda_{2}=\lambda_{3}=0.5, k_{1}=k_{2}=k_{3}=2$.

$$
\begin{aligned}
& \frac{\partial v}{\partial t}+u \frac{\partial v}{\partial x}+v \frac{\partial v}{\partial y}+w \frac{\partial v}{\partial z}=-g_{y}-\frac{1}{\rho} \frac{\partial p}{\partial y}-\ddot{y}_{C} \\
& -(\ddot{\gamma} x-\ddot{\alpha} z)-\left(2 \dot{\beta} \dot{\gamma}-\dot{\alpha}^{2}-\dot{\gamma}^{2}\right) y-2(\dot{\gamma} u-\dot{\alpha} w)-\left(\dot{\gamma} u_{t}-\dot{\alpha} w_{t}\right) \\
& \frac{\partial w}{\partial t}+u \frac{\partial w}{\partial x}+v \frac{\partial w}{\partial y}+w \frac{\partial w}{\partial z}=-g_{z}-\frac{1}{\rho} \frac{\partial p}{\partial z}-\ddot{z}_{C} \\
& -(\ddot{\alpha} y-\ddot{\beta} x)-\left(2 \dot{\alpha} \dot{\gamma}-\dot{\alpha}^{2}-\dot{\beta}^{2}\right) z-2(\dot{\alpha} v-\dot{\beta} u)-\left(\dot{\alpha} v_{t}-\dot{\beta} u_{t}\right)
\end{aligned}
$$

where $u, v$ and $w$ are the relative velocity components in $x, y$ and $z$ directions, $u_{t}, v_{t}$ and $w_{t}$ are velocity components of the tank in $x, y$ and $z$ directions, $\ddot{x}_{C}, \ddot{y}_{C}$ and $\ddot{z}_{C}$ are the relative acceleration components of the tank in $x, y$ and $z$ directions; ( $\dot{\alpha}, \dot{\beta}, \dot{\gamma})$ and $(\ddot{\alpha}, \ddot{\beta}, \ddot{\gamma})$ are the corresponding angular velocities and accelerations about the $x, y$ and $z$-axes respectively, $p$ is the pressure, $\rho$ is the fluid density, and $g_{x}, g_{y}$ and $g_{z}$ are the components of the acceleration due to gravity.

The continuity equation for incompressible flow is

$$
\frac{\partial u}{\partial x}+\frac{\partial v}{\partial y}+\frac{\partial w}{\partial z}=0
$$

the kinematic boundary condition on the free surface is

$$
\frac{\partial h}{\partial t}+u \frac{\partial h}{\partial x}+w \frac{\partial h}{\partial z}=v
$$


and the dynamic free surface condition is $p=0$. The general forms of boundary conditions at the solid walls are

$$
\frac{1}{\rho} \frac{\partial p}{\partial x}=-g_{x}-\ddot{x}_{C}-(\ddot{\beta} z-\ddot{\gamma} y)-\left(2 \dot{\alpha} \dot{\beta}-\dot{\beta}^{2}-\dot{\gamma}^{2}\right) x-2(\dot{\beta} w-\dot{\gamma} v)
$$

$\frac{1}{\rho} \frac{\partial p}{\partial y}=-g_{y}-\ddot{y}_{C}-(\ddot{\gamma} x-\ddot{\alpha} z)-\left(2 \dot{\beta} \dot{\gamma}-\dot{\alpha}^{2}-\dot{\gamma}^{2}\right) y-2(\dot{\gamma} u-\dot{\alpha} w)$

$\frac{1}{\rho} \frac{\partial p}{\partial z}=-g_{z}-\ddot{z}_{C}-(\ddot{\alpha} y-\ddot{\beta} x)-\left(2 \dot{\alpha} \dot{\gamma}-\dot{\alpha}^{2}-\dot{\beta}^{2}\right) z-2(\dot{\alpha} v-\dot{\beta} u)$

Taking partial derivatives of Eqs. (1), (2) and (3) with respect to $x, y$ and $z$ respectively, and summing the results, one can obtain the following poisson equation which is used to solve for the pressure.

$$
\begin{aligned}
& \frac{\partial^{2} p}{\partial x^{2}}+\frac{\partial^{2} p}{\partial y^{2}}+\frac{\partial^{2} p}{\partial z^{2}}=-\rho \frac{\partial}{\partial x}\left(u \frac{\partial u}{\partial x}+v \frac{\partial u}{\partial y}+w \frac{\partial u}{\partial z}\right) \\
& -\rho \frac{\partial}{\partial y}\left(u \frac{\partial v}{\partial x}+v \frac{\partial v}{\partial y}+w \frac{\partial v}{\partial z}\right)-\rho \frac{\partial}{\partial z}\left(u \frac{\partial w}{\partial x}+v \frac{\partial w}{\partial y}+w \frac{\partial w}{\partial z}\right) \\
& \left.-2 \rho[\dot{\alpha} \dot{\beta}+\dot{\beta} \dot{\gamma}+\dot{\alpha} \dot{\gamma})-\left(\dot{\alpha}^{2}+\dot{\beta}^{2}+\dot{\gamma}^{2}\right)\right] \\
& -2 \rho\left[\dot{\alpha}\left(\frac{\partial v}{\partial z}-\frac{\partial w}{\partial y}\right)+\dot{\beta}\left(\frac{\partial w}{\partial x}-\frac{\partial u}{\partial z}\right)+\dot{\gamma}\left(\frac{\partial u}{\partial y}-\frac{\partial v}{\partial x}\right)\right]
\end{aligned}
$$

\section{Coordinate Transformation}

Many finite difference and volume methods have been reported to solve the free surface displacement of sloshing fluid in tanks. Among these methods, SURF, MAC and VOF are the most well-known methods. The SURF scheme assumes a single valued surface profile and is potentially able to deal with a uniform representation of large free surface waves and even for overturning inception. The famous MAC method is a Largrangian concept and can treat overturning waves and reentry inception with a simple logic. While the VOF method tracks the volume occupied by the fluid rather than the free surface and the method is the most popular method used in the literatures. All the above methods could properly calculate the instant free surface displacement but they all need complicate computer programming in treating the time varied free surface boundary and updating computational meshes. Alternately, in the present study, the time-varied free surface boundary is transformed to a time-independent free surface in the $x^{*}-y^{*}-z^{*}$ domain and no boundary tracing is needed during the calculation. A single value height function is assumed and is evaluated by solving the kinematic free surface condition. The irregular boundary, including the time-varying fluid surface, non-vertical walls and non-horizontal bottom, can be mapped onto a cube by the proper coordinate transformations (Hung and Wang [17], Hung and Chen, [16]) as follows:

$$
\begin{gathered}
x^{*}=\frac{x-b_{1}(y, z)}{b_{2}(y, z)-b_{1}(y, z)} \\
y^{*}=1-\frac{y+d(x, z)}{h(x, z, t)} \\
z^{*}=\frac{z-b_{3}(x, y)}{b_{4}(x, y)-b_{3}(x, y)}
\end{gathered}
$$

where the instantaneous water surface, $h(x, z, t)$, is a single-valued function measured from the tank bottom, $d(x, z)$ represents the vertical distance between the still water surface and the tank bottom, $b_{1}$ and $b_{2}$ are horizontal distances from the $\mathrm{x}$-axis to the west and east walls respectively, and $b_{3}$ and $b_{4}$ are horizontal distances from the $\mathrm{z}$-axis to the north and south walls respectively, see Fig. 1. Through Eqs. (10) to (12), one can map the west wall to $x^{*}=0$ and east wall to $x^{*}=1$, the north wall to $z^{*}=0$ and south wall to $z^{*}=1$, the free surface to $y^{*}=0$ and the tank bottom to $y^{*}=1$. In this way the computational domain is transformed to a fixed unit cubic domain. The main advantage of these transformations is to map a wavy and time-dependent fluid domain onto a time-independent unit cubic domain. In this way re-meshing due to the wavy free surface is unnecessary. In addition, the mapping implicitly deals with the free surface motion, and avoids the need to calculate the free surface velocity components explicitly. Extrapolations are unnecessary, and free surface smoothing by means of a spatial filter is not required.

The coordinates $\left(x^{*}, y^{*}, z^{*}\right)$ can be further transformed such that the layer near the boundary is stretched to capture sharp local velocity gradients. The following exponential functions provide these stretching transformations:

$$
\begin{aligned}
& X=\lambda_{1}+\left(x^{*}-\lambda_{1}\right) e^{k_{1} x^{*}\left(x^{*}-1\right)} \\
& Y=\lambda_{2}+\left(y^{*}-\lambda_{2}\right) e^{k_{2} y^{*}\left(y^{*}-1\right)} \\
& Z=\lambda_{\in 3}+\left(z^{*}-\lambda_{3}\right) e^{k_{3} z^{*}\left(z^{*}-1\right)}
\end{aligned}
$$

The constants $k_{2}$ and $\lambda_{2}$ control the mesh size near the free surface and tank bottom. The constants $k_{1}, \lambda_{1}, k_{3}$ and $\lambda_{3}$ similarly control the mesh in the horizontal directions. Thus, the geometry of the flow field and the meshes in the computational domain (X-Y-Z system) become time-independent throughout the computational analysis. Note the fluid domain in the X-Y-Z system remains a unit cube. In order to increase the accuracy of the computation, especially near the boundary, the stretch factors, $\lambda_{1}=\lambda_{2}=\lambda_{3}=0.5$ and $k_{1}=k_{2}=k_{3}=2$, are used in the present study 


\section{Dimensionless Equations}

The dimensional parameters are normalized or non-dimensionalised using the following equations.

$$
\begin{aligned}
& U=\frac{u}{\sqrt{g d_{0}}} \quad V=\frac{v}{\sqrt{g d_{0}}} \quad W=\frac{w}{\sqrt{g d_{0}}} \quad P=\frac{p}{\sqrt{\rho g d_{0}}} \\
& T=t \sqrt{\frac{g}{d_{0}}} \quad H=\frac{h-d_{0}}{d_{0}} \quad X_{c}=\frac{x_{c}}{d_{0}} \quad Y_{c}=\frac{y_{c}}{d_{0}} \quad Z_{c}=\frac{z_{c}}{d_{0}} \\
& \Theta_{\alpha}=\frac{\alpha}{2 \pi} \quad \Theta_{\beta}=\frac{\beta}{2 \pi} \quad \Theta_{\gamma}=\frac{\gamma}{2 \pi} \quad \Theta_{\alpha T}=\frac{\dot{\alpha}}{2 \pi} \sqrt{\frac{d_{0}}{g}} \\
& \Theta_{\beta T}=\frac{\dot{\beta}}{2 \pi} \sqrt{\frac{d_{0}}{g}} \quad \Theta_{\gamma T}=\frac{\dot{\gamma}}{2 \pi} \sqrt{\frac{d_{0}}{g}} \quad \Theta_{\alpha T T}=\frac{\ddot{\alpha}}{2 \pi} \frac{d_{0}}{g} \\
& \Theta_{\beta T T}=\frac{\ddot{\beta}}{2 \pi} \frac{d_{0}}{g} \quad \Theta_{\gamma T T}=\frac{\ddot{\gamma}}{2 \pi} \frac{d_{0}}{g}
\end{aligned}
$$

where $d_{0}$ is the undisturbed fluid depth (assumed constant for the remainder of this paper), and $x_{c}, y_{c}$ and $z_{c}$ are the tank displacements in the $x, y$ and $z$ directions, respectively.

With the aforementioned transformations and dimensionless variables, Eqs. (1)-(9) can be written in dimensionless form. For example the momentum equation in the $\mathrm{x}$-direction is

$$
\begin{aligned}
U_{T} & +\left(C_{10} C_{13} U_{X}+C_{11} C_{14} U_{Y}+C_{12} C_{15} U_{Z}\right)+C_{1} C_{13} U U_{X} \\
& +C_{2} C_{14} U U_{Y}+C_{3} C_{15} U U_{Z}+C_{4} C_{13} V U_{X} \\
& +C_{5} C_{14} V U_{Y}+C_{6} C_{15} V U_{Z}+C_{7} C_{13} W U_{X}+C_{8} C_{14} W U_{Y} \\
& +C_{9} C_{15} W U_{Z}=-G_{\aleph}-\left(C_{1} C_{13} P_{X}+C_{2} C_{14} P_{Y}+C_{3} C_{15} P_{Z}\right) \\
& -X_{C T T}-\frac{2 \pi}{d_{0}}\left(z \Theta_{\beta_{T T}}-y \Theta_{\gamma_{T T}}\right) \\
& -\frac{4 \pi^{2}}{d_{0}}\left(2 \Theta_{\alpha T} \Theta_{\beta T}-\Theta_{\beta T}^{2}-\Theta_{\gamma T}^{2}\right) \aleph \\
& -4 \pi\left(\Theta_{\beta T} W-\Theta_{\gamma T} V\right)
\end{aligned}
$$

The dimensionless forms of the other equations are listed in Appendix A. In Eq. (17), $C_{1}-C_{15}$ are coefficients that arise from the coordinate transformations and can be found in [26]. $P_{X}$ denotes a partial derivative of $P$ with respect to $\mathrm{X}, U_{\mathrm{T}}$ is the partial derivative of $U$ with respect to dimensionless time $T$, and so $X_{C T T}, Y_{C T T}$ and $Z_{C T T}$ are dimensionless ground accelerations in the $\mathrm{X}, \mathrm{Y}$ and $\mathrm{Z}$ directions. All other terms have similar meanings.

\section{COMPUTATIONAL ALGORITHM}

In this three-dimensional analysis, the fluid flow is solved in a unit cubic mesh in the transformed flow domain. All computations use the dimensionless equations in the $\mathrm{X}-\mathrm{Y}-\mathrm{Z}$ coordinate system. Central difference approximations are used for the space derivatives, except at the boundary where forward or backward differences are employed. A staggered grid system is used in the analysis. That is, the pressure $P$ is defined at the centre of a finite difference grid cell (of dimensions $\Delta X, \Delta Y, \Delta Z$ ), whereas the velocity components $U, V$ and $W$ are calculated $0.5 \Delta X, 0.5 \Delta Y$ and $0.5 \Delta Z$ behind, above or backward of the cell centre.

\section{Finite Difference Method}

The Crank-Nicholson second order finite difference scheme and the Gauss-Seidel point successive over-relaxation iterative procedure are used to calculate the velocity and pressure, respectively. The numerical scheme is described below. When the dimensionless momentum equations, ex: Eqs. (17) are considered to be balanced at time $T=(n+1 / 2) \Delta T$, one can express them in the following finite-difference form.

$$
\begin{aligned}
& U_{i, j, k}^{n+1}=U_{i, j, k}^{n}-\Delta T\left(\wp_{i, j, k}+P_{i, j, k}^{X}\right) \\
& V_{i, j, k}^{n+1}=V_{i, j, k}^{n}-\Delta T\left(\Re_{i, j, k}+P_{i, j, k}^{Y}\right) \\
& W_{i, j, k}^{n+1}=W_{i, j, k}^{n}-\Delta T\left(\aleph_{i, j, k}+P_{i, j, k}^{Z}\right) \\
& H_{i, j, k}^{n+1}=H_{i, j, k}^{n}-\Delta T\left(\mathfrak{J}_{i, j, k}+V_{i, j, k}\right)
\end{aligned}
$$

In these equations, the superscript $\mathrm{n}$ represents the time index (i.e. $T=n \Delta T$ ). The terms without a superscript are at $T=(n+1 / 2) \Delta T$. The velocity components at $T=(n+1 / 2) \Delta T$ can be approximated as the averages of the values at $n \Delta \mathrm{T}$ and $(n+1) \Delta T$. All of the terms on the right-hand side of Eqs. (18), (19) and (20) are applied at the same nodes as $U_{i, j, k}, V_{i, j, k}$ and $W_{i, j, k}$. The terms $P_{i, j, k}^{X}, P_{i, j, k}^{Y}$ and $P_{i, j, k}^{Z}$ are the corresponding pressure gradients in the $\mathrm{X}, \mathrm{Y}$ and $\mathrm{Z}$ directions, respectively. The terms $\wp_{i, j, k}, \Re_{i, j, k}$ and $\aleph_{i, j, k}$ contain all of the remaining terms in Eq. (17), including the finite-difference expressions for the convective acceleration, and the terms related to surge, sway, heave, pitch, roll and yaw motions. In Eq. (21), $\mathfrak{J}_{i, j, k}$ is the non-linear term of the equation.

The pressure is evaluated by solving the Poisson equation. For $T=(n+1 / 2) \Delta T$, one can express the finite-difference equation in the following form

$$
P_{i, j, k}=\frac{\Psi}{a_{i, j, k}}\left[\Pi_{i, j, k}+\Omega_{i, j, k}\right]+(1-\Psi) P_{i, j, k}^{*}
$$

in which $a_{i, j, k}$ is the sum of the coefficients of pressure $P_{i, j, k}$, $\Psi$ is the relaxation parameter, and $P_{i, j, k}^{*}$ is the previous iterated pressure. The relaxation parameter $\Psi$ is chosen to be 0.5 in the present study and much iteration time is reduced. The 


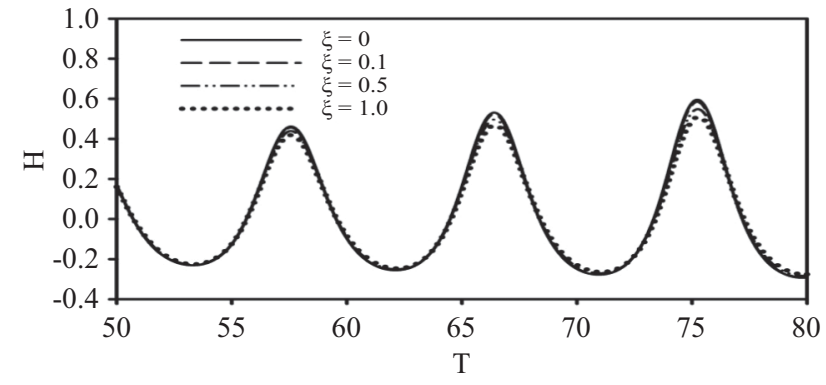

Fig. 2. The damping influence of weight parameter $\xi$. The wave history at the tank's corner A under coupled Surge-Sway motion. The ratio $d_{0} / \mathrm{L}=d_{0} / \mathrm{B}=0.25, \mathrm{~A} / \mathrm{L}=0.005$. $\omega_{\mathrm{x}}=\omega_{\mathrm{z}}=0.97 \omega_{1}$. Excitation angle $\theta=5^{\circ}$.

terms $\Pi_{i, j, k}$ represent the finite-difference expressions of the pressure gradient and $\Omega_{i, j, k}$ the finite-difference expressions of the nonlinear convective accelerations and the term related to tank motion (surge, sway, heave, roll, pitch and yaw). The superscript $(n+1 / 2)$, for $P_{i, j, k}, \Pi_{i, j, k}$ and $\Omega_{i, j, k}$ is also omitted here. The detailed finite difference expressions for $P_{i, j, k}, \Pi_{i, j, k}$ and $\Omega_{i, j, k}$ are tedious, and are, therefore, omitted from the text. Once the pressure field has been solved by iteration, the velocity components $U_{i, j, k}^{n+1}, V_{i, j, k}^{n+1}$ and $W_{i, j, k}^{n+1}$ can be calculated from equations, Eqs. (18), (19) and (20). The instantaneous water surface profile $H_{i, j, k}^{n+1}$ can be calculated from Eq. (21). In the present study, only the single-valued profile is assumed on the free surface. This assumption is not valid when splash or wave breaking occurs.

The difference approximation of the convective terms of momentum equations are very sensitive to numerical solution and the second-order central difference upwind scheme (Hirt et al., [15]) is adopted in the present numerical scheme. That is, using a weight parameter $\xi$, the second-order central difference and first order upwind scheme can be written as

$\left(\frac{\partial f}{\partial s}\right)=\frac{1+\xi \operatorname{sgn}\left(f_{i}\right)}{2}\left(\frac{f_{i}-f_{i-1}}{\Delta s}\right)+\frac{1-\xi \operatorname{sgn}\left(f_{i}\right)}{2}\left(\frac{f_{i+1}-f_{i}}{\Delta s}\right)$

where $f$ can be any function of parameter such as velocity $u, v$, and $w, \Delta s$ means the mesh size and $\operatorname{sgn}\left(f_{i}\right)$ is the sign function. This scheme is also used to solve the wave elevation from the kinematic free surface boundary condition. As $\xi$ is equal to 1 or 0 , Eq. (23) becomes $1^{\text {st }}$ order upwind scheme or central finite difference, respectively. The value of $\xi$ is determined by a parametric study. As plotted in Fig. 2, the larger $\xi$ is, the greater numerical damping. And the weight parameter $\xi$ is, therefore, set to be 0.1 in the present numerical model after a parametric test.

The accuracy of the numerical results significantly depends on the spatial grid resolution and the selected time step. Thus, it is important to verify the appropriate time step for a particular spatial grid before large numerical problems are simu- lated. The numerical errors can be reduced if the time step is restricted by the condition given in Eq. (24)

$$
\Delta t<\min \left\{\frac{\Delta x_{\min }}{\left|u_{i, j, k}\right|}, \frac{\Delta y_{\min }}{\left|v_{i, j, k}\right|}, \frac{\Delta z_{\min }}{\left|w_{i, j, k}\right|}\right\}
$$

Eq. (24) implies that a fluid particle cannot move more than one cell in a single time step.

\section{Iterative Procedure}

The finite-difference equations mentioned above can be used to solve for the wave field and internal flow field as the tank is subject to external forcing. The most difficult part of the present study is to calculate the coefficients of pressure, $a_{i, j, k}$. A new iterative procedure similar to SIMPLEC algorithm (Van Doormaal and Raithby, [25]) is developed and the computational time reduces at least a half of that by implementing the original iterative procedure reported by Chen and Nokes [6]. The detailed implicit iterative solution procedure employed here is given below. The convergence criterion for the iteration of $U, V, W$ and $P$ is $10^{-5}$, while for $\mathrm{H}$ it is set to $10^{-7}$.

Implicit iterative processes:

1. Specify the initial condition

2. Update forcing condition (tank motion)

3. Calculate coefficients $\mathrm{C}_{1}-\mathrm{C}_{15}$ and calculate the coefficient of pressure, $a_{i, j, k}$.

4. Calculate $\wp_{i, j, k}, \Re_{i, j, k}$ and $\aleph_{i, j, k}$.

5. Substitute the results of step 4 into Eq. (22) in order to calculate $\Omega_{i, j, k}$.

6. Using the boundary conditions on pressure, calculate the terms $P_{i, j, k}^{X}, P_{i, j, k}^{Y}$ and $P_{i, j, k}^{Z}$ in order to calculate $\Pi_{i, j, k}$.

7. Calculate $U_{i, j, k}, V_{i, j, k}$ and $W_{i, j, k}$ from Eqs. (18), (19) and (20), respectively.

8. Calculate $P_{i, j, k}$ from Eq. (22) and then recalculate the terms $P_{i, j, k}^{X}, P_{i, j, k}^{Y}$ and $P_{i, j, k}^{Z}$.

9. Recalculate new $U_{i, j, k}, V_{i, j, k}$ and $W_{i, j, k}$ from Eqs. (18), (19) and (20), respectively.

10. Average the velocities calculated from steps 7 and 9 and then get new $U_{i, j, k}, V_{i, j, k}$ and $W_{i, j, k}$.

11. Repeat steps 6 and 9 at least 2 times, then check for convergence; that is, check if $\left|P^{k+1}-P^{k}\right|<10^{-5},\left|U^{k+1}-U^{k}\right|<$ $10^{-5},\left|V^{k+1}-V^{k}\right|<10^{-5}$ and $\left|W^{k+1}-W^{k}\right|<10^{-5}$ in which $k$ represents the iteration number. If convergence is not reached, repeat steps 4-10.

12. Calculate $H_{i, j, k}$ from Eq. (21) and check that $\left|H^{k+1}-H^{k}\right|<$ $10^{-7}$. If the convergence has not been reached, go to step 3 and update the coefficients relating to $H$.

13. If $H$ has converged then goes to step 2 and begin the next time step. 


\section{RESULTS AND DISCUSSION}

The wave motion in a three dimensional tank is considerably more complicated than that in a two dimensional tank. For a three dimensional tank traveling in a vehicle on a highway, or on a ship on the sea, the angle of the excitation motion can change randomly in time and a small perturbation in excitation angle might significantly affect the sloshing response of the fluid in the tank. As indicated earlier, if the tank is excited by an earthquake, the duration of which may be less than 60 seconds, then a steady state response is unlikely to be achieved during the time span of the excitation and, therefore, the transient motion will be of most importance. Therefore, our main emphasis in this study is to focus on the transient fluid motion caused by harmonic excitation with various excitation frequencies and angles.

The natural modes of a 3-D tank with square base can be obtained by solving the linearized natural sloshing standing wave problem. The angular frequencies, $\omega_{i, j}$ of these natural modes, are given in Eq. (25)

$$
\begin{aligned}
& \lambda_{i, j}=\pi \sqrt{i^{2}+j^{2}} \\
& \omega_{i, j}^{2}=g \lambda_{i, j} \tanh \left(\lambda_{i, j} d_{0}\right)
\end{aligned}
$$

where $i, j$ are the natural mode's components of $\mathrm{x}$ - and $\mathrm{z}$-axes and $\omega_{i, j}^{2}$ are natural frequencies of the 3-D tank. While six degrees of freedom for the tank motion can be included in the present model, simulations are only presented for coupled surge-sway motion. Extensive simulations have been made with the model, but the simulations for cases with strong shallow-fluid sloshing and large excitation amplitude were failed to satisfactorily converge and thus these simulations are beyond the current capability of our model.

\section{Benchmark Tests}

In order to validate the accuracy of our model, the simulated results are compared with those reported in the literature. Fig. 3(a) shows the sloshing displacement in the corners A and $\mathrm{B}$ of a tank with depth $\left(d_{0}\right) /$ breadth $(\mathrm{L})$ ratio $=0.25$ when the tank is diagonally excited by a ground motion with an excitation displacement $\mathrm{A}=0.0093 \mathrm{~L}$, and an excitation frequency equals to $0.99 \omega_{1}\left(\omega_{1}=\omega_{1,0}\right.$, the first natural frequency of the tank). The results reported by Kim [20] are also shown in the figure and the agreement is satisfactory. Kim used SURF scheme to solve for the kinematic boundary condition and used a numerical filtering of five-point formula to avoid the stability due to saw-tooth waves. In the present study, we use simple mapping functions to remove the time-dependence of free surface. In this way, re-meshing due to the wavy free surface is unnecessary and the calculation of the free surface components explicitly is avoided. Besides, the present numerical scheme can achieve accurate numerical results based on a relatively coarse meshes $(20 \times 10 \times 20)$ compared with Kim's $(30 \times 30 \times 30)$ meshes. Both simulation time and memory are, therefore, dramatically reduced in this study.

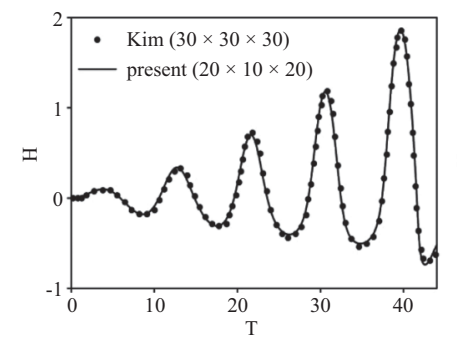

(a)

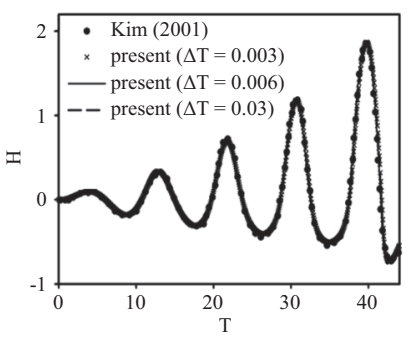

(b)
Fig. 3. The wave history on corner $A$ of the tank under diagonal motion, $d_{0} / \mathrm{L}=d_{0} / \mathrm{B}=0.25, \mathrm{~A}$ (excitation displacement) $/ \mathrm{L}=0.0093, \omega_{x}=$ $\omega_{z}=0.99 \omega_{1}$. (a) the comparison between Kim's (mesh: $30 \times 30 \times$ 30) results and the present results $(20 \times 10 \times 20)(b)$ the influence of different time step with the same mesh number $(20 \times 10 \times 20)$.

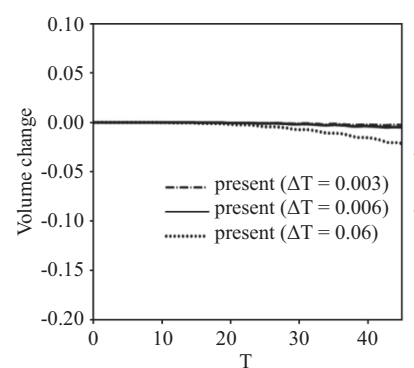

(a)

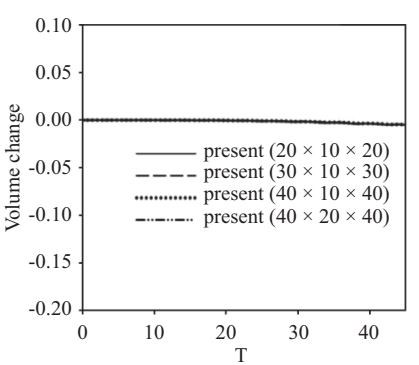

(b)
Fig. 4. The conservation of fluid mass during liquid sloshing under (a) various time steps and (b) various mesh arrangements.

Also shown in Fig. 3 are the parametric tests for different time steps. Fig. 3(b) depicts fixed mesh numbers $(20 \times 10 \times 20)$ with various time steps. As shown in the figure, the influence of time steps on wave elevation is insignificant.

We further check the mass balance of the numerical simulation. The continuity of fluid mass is shown in Fig. 4. Fig. 4(a) presents the numerical errors influenced by various time step and the results show that the numerical errors increase with the magnification of the time step. The numerical errors influenced by mesh number are shown in Fig. 4(b) and it shows that numerical errors are all negligible small for various mesh sizes. For excitation frequency far away from the first fundamental frequency, larger time step $(\Delta T=0.03)$ can be applied in the simulation. Whereas, for near-resonant excitation, smaller time step $(\Delta T=0.006 \sim 0.003)$ is chosen so as to reduce the numerical errors in the present study. As the parametric study mentioned above, the selection of a grid resolution of $\Delta X=$ $\Delta Z=0.05, \Delta T=0.006$, are used in all the simulations in the present study.

Fig. 5 compares the sloshing displacements of fluid in a tank excited by a heave (vertical) motion with those reported by Frandsen [13] and again the agreement is very good. Besides, the present numerical results are further validated by comparing with experimental and analytical results reported by Faltinsen et al. [9]. The comparisons (Fig. 6) show, once again, excellent agreements among numerical, experimental and analytical results. 


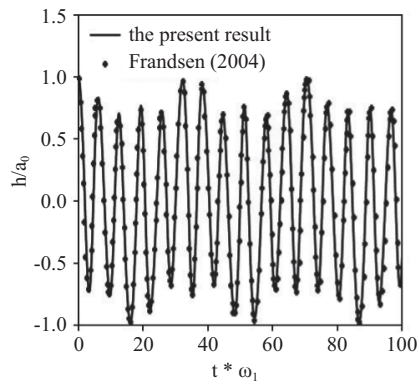

(a)

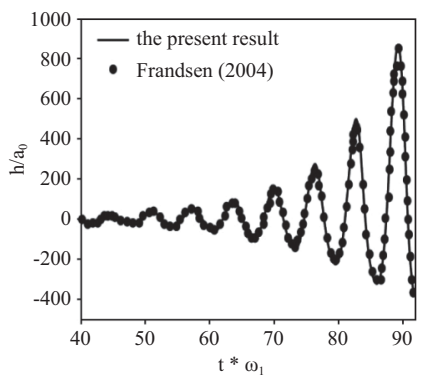

(b)
Fig. 5. The wave history on tank's corner (corner A) under Heave (vertical) motion, $d_{0} / \mathrm{L}=d_{0} / \mathrm{B}=0.5$. (a) Tank's displacement $\mathrm{A} / \mathrm{L}=$ $0.272, \omega_{y}=0.8 \omega_{1}$; (b) $\mathrm{A} / \mathrm{L}=0.272, \omega_{y}=2 \omega_{1}, \mathrm{a}_{0}$ : the initial perturbation $=0.0001215 \mathrm{~m}$ (Frandsen, 2004).

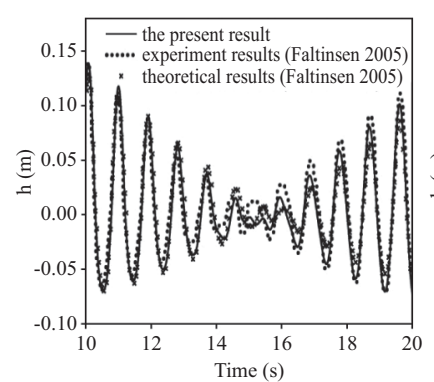

(a)

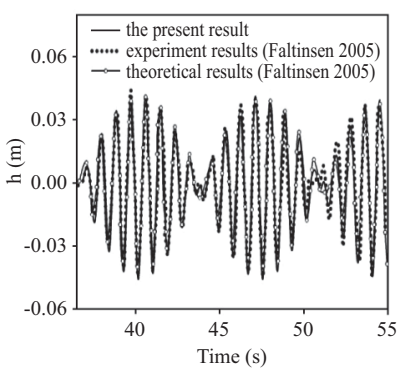

(b)
Fig. 6. The comparison of the present result with the experimental and theoretical results reported by Faltinsen et al. $(2005), d_{0} / \mathrm{L}=d_{0} / \mathrm{B}=$ 0.5. Excitation displacement $\mathrm{A} / \mathrm{L}=\mathbf{0 . 0 0 7 8}$, (a) surge motion, $\omega_{x}=$ $1.037 \omega_{1}$; (b) diagonal motion, $\omega_{x}=\omega_{z}=1.115 \omega_{1}$.

\section{Horizontal Ground Motion}

For practical engineering applications, for example a tank excited by a real earthquake, the ground motion will be a complex combination of surge, sway, heave, pitch, yaw and roll. Initially, if we focus on the horizontal excitation only, the tank, in general, would not be excited exactly in either the surge (x-) or sway (z-) directions, but rather it will be a coupled surge and sway motion, and the excitation direction may vary with time. In this paper, we define $\theta$ as the excitation angle of the horizontal ground motion. For longitudinal excitation, surge motion with $\theta=0^{\circ}$, Faltinsen et al. [8] reported four different kinds of waves - planar waves $\left(\omega_{x} / \omega_{1}=0.92\right.$; $\mathrm{h}=0.508)$, swirling waves $\left(\omega_{x} / \omega_{1}=1.011\right)$, irregular waves $\left(\omega_{x} / \omega_{1}=0.945\right)$ and square-like waves $\left(\omega_{x} / \omega_{1}=0.98\right)$. Here $\omega_{x}$ is the excitation frequency of longitudinal excitation and $\omega_{1}=\omega_{1,0}$ from Eq. (25). When the tank was excited by diagonal motion $\left(\theta=45^{\circ}\right)$, the same four kinds of waves might be expected, but the stable square-like waves and swirling waves were seldom seen in their experimental measurements. Visual observations made by Faltinsen et al. [8] showed that three dimensional waves are observed only when the tank was under near resonant excitation. When the excitation was away from the lowest natural frequency, the sloshing responses were

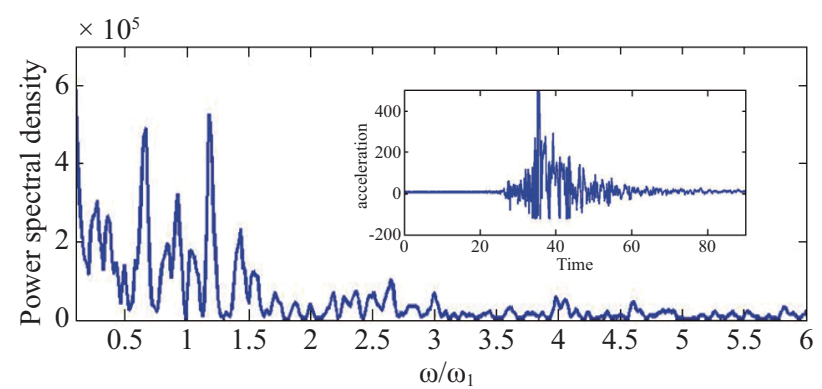

Fig. 7. The spectral analysis of 921, 1999 ChiChi earthquake. Subfigure: the time history of acceleration $\left(\mathrm{m} / \mathrm{s}^{2}\right) . \omega_{1}$ : the fundamental natural frequency of a tank with $d_{0} / \mathrm{L}=d_{0} / \mathrm{B}=0.25$.

basically two-dimensional longitudinal excitation or diagonal sloshing (under diagonal excitation). In the present study, longitudinal excitation and diagonal excitation and a wide range of excitation frequencies, are simulated.

In this section, the tank is excited by surge-sway motion with various excitation frequencies. The tank is rigid with a square base and an undisturbed fluid depth of $d_{0} / \mathrm{L}=0.25$. The amplitude of the ground displacement is $0.005 \mathrm{~L}$. The spectral analysis of a typical earthquake record (921 Chi-Chi earthquake, Taiwan 1999), presented in Fig. 7, indicates that the majority of the energy resides within the frequency range of $0.2 \omega_{1}$ to $2.5 \omega_{1}$. Therefore, in the present study, excitation frequencies from $0.2 \omega_{1}$ to $2.3 \omega_{1}$ are used. The different types of sloshing wave that arise with different excitation frequencies and excitation angles are discussed separately in the following subsections.

\section{Diagonal Excitation}

For non-resonant excitation, a diagonal wave, in which the wave sloshing occurred only in the diagonal direction of the tank, was first identified by Miles [21]. In Faltinsen's studies the diagonal wave occurred when the tank was excited by frequencies far from the first natural frequency. Fig. 8 shows pattern of the wave history of the fluid sloshing in a tank excited by diagonal ground motion $\left(\theta=45^{\circ}\right)$ with an excitation frequency of $\omega=0.8 \omega_{1}$. The contour of the surface elevation, Fig. 8(b) shows clearly that the waves are traveling in the diagonal direction. For a fixed excitation angle of $\theta=45^{\circ}$, the diagonal waves also can be found when the tank is excited at other excitation frequencies. However, as the tank is accelerated at other angles, the diagonal wave no longer occurs. Instead, the waves are found to slosh in the same direction as the excitation motion. We can define this type of wave as a 'single-directional' wave. Fig. 9 presents the surface contour profiles of single-directional waves with various excitation angles, and a fixed excitation frequency of $0.4 \omega_{1}$. The single-directional waves have been reported in the literature but all the earlier studies have been limited to a tank undergone longitudinal forcing ( $\operatorname{surge} \theta=0^{\circ}$ ) or diagonal forcing $(\theta=$ $45^{\circ}$ ). 


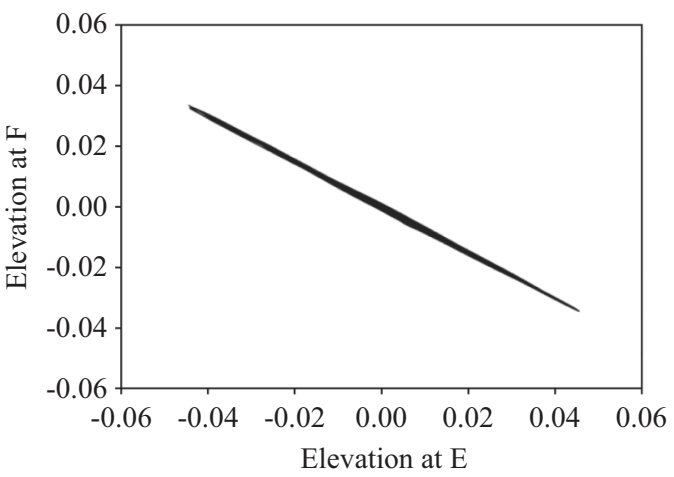

(a)

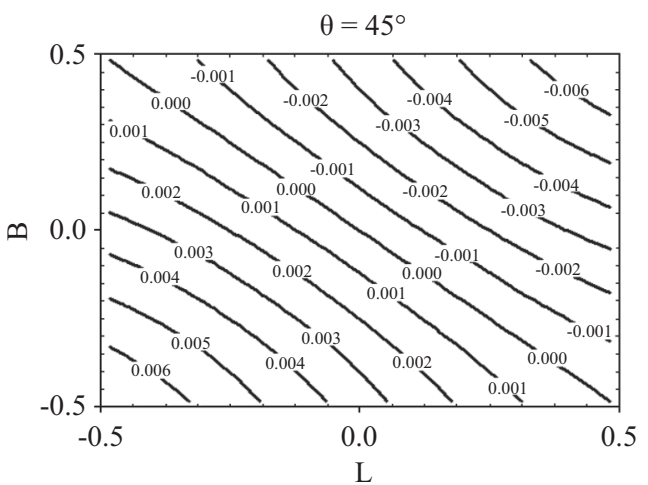

(b)

Fig. 8. The diagonal waves. The tank is vibrated in diagonal $\left(\theta=45^{\circ}\right)$ direction during transient period. The excitation frequency $=$ $0.8 \omega_{1}$. (a) the pattern of diagonal waves (b) the contours of free surface.
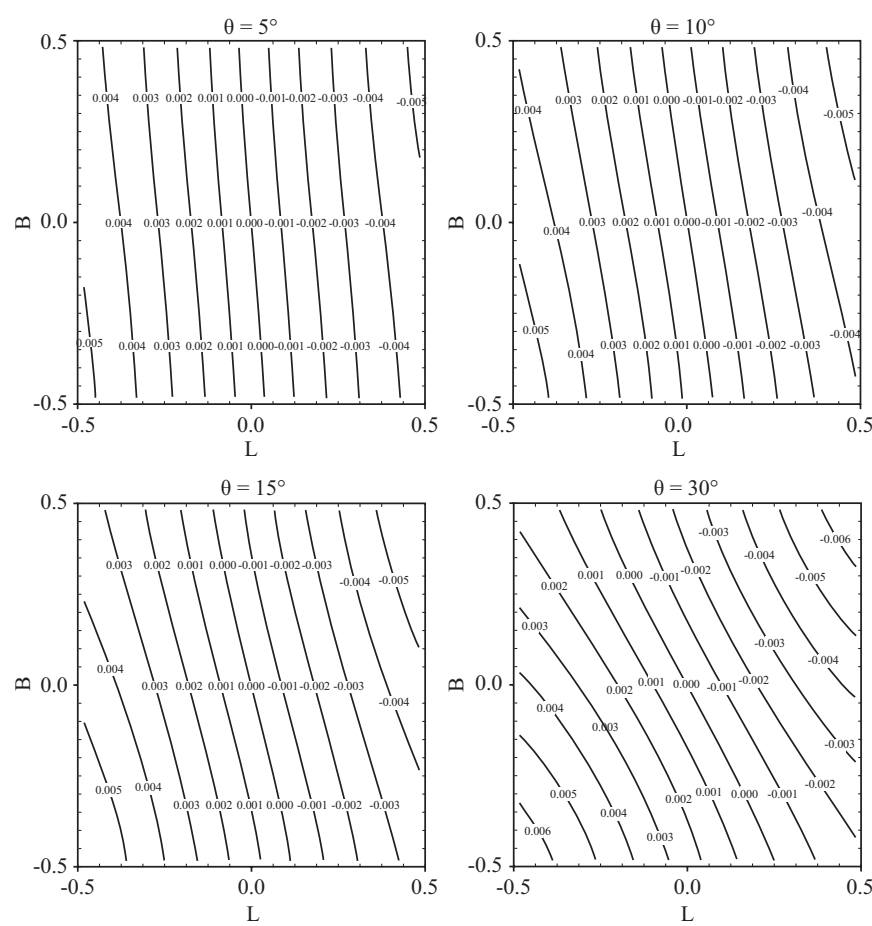

Fig. 9. The free surface contours of single-directional waves with various excitation angles. The excitation frequency is equal to $0.4 \omega_{1}$.

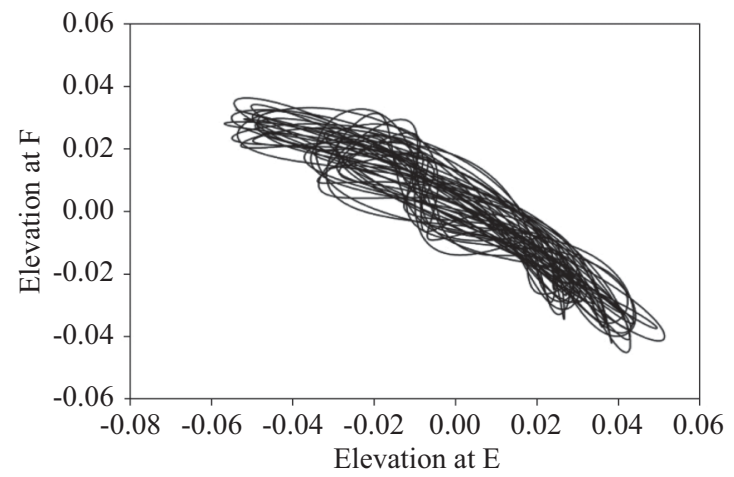

(a)

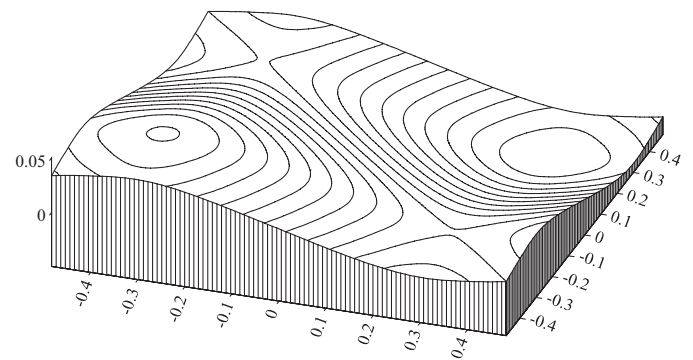

(b)

Fig. 10. The square-like waves. The tank is vibrated at $\theta=45^{\circ}$ during transient period with excitation frequency $\omega=1.5 \omega_{1}$. (a) the pattern of square-like waves (b) the contours of free surface.

\section{Frequently Obtained Square-like Waves and Chaotic Waves}

Faltinsen et al. [8] theoretically predicted that square-like waves can co-exist with planar waves (the sloshing waves under longitudinal forcing), although they found the squarelike waves were rarely observed, and were only confirmed in one experimental series of longitudinal excitation at $\omega_{x} / \omega_{1}=$ 0.98. The classification of square-like waves is, therefore, omitted in their later articles. The phenomenon of square-like waves corresponds to waves traveling primarily on two opposite sides of the tank. In previous studies (e.g. Faltisen et al., [8]) square-like waves were discussed for a tank excited at near resonant conditions. However, in the present study, square-like waves can be found when the tank is excited at non-resonant frequencies. Fig. 10 presents results for a tank excited by diagonal forcing with an excitation frequency of $1.5 \omega_{1}$. The relationship between the surface elevation at points $\mathrm{E} \& \mathrm{~F}$ and the surface elevation contours are shown in Figs. 10 (a) and (b), respectively. The surface elevation contours in Fig. 10(b) clearly shows square-like waves that are quite different from the so-called diagonal sloshing or single-directional sloshing previously considered.

Fig. 11 presents the free surface contour of square-like waves in a tank accelerated at various excitation angles with the same excitation frequency used in Fig. 10. As shown in the figure, the square-like form becomes more pronounced as the angle increases. The terraced planes are clearly seen for the cases of the tank under diagonal excitation. Figs. 11 (e) and (f) 

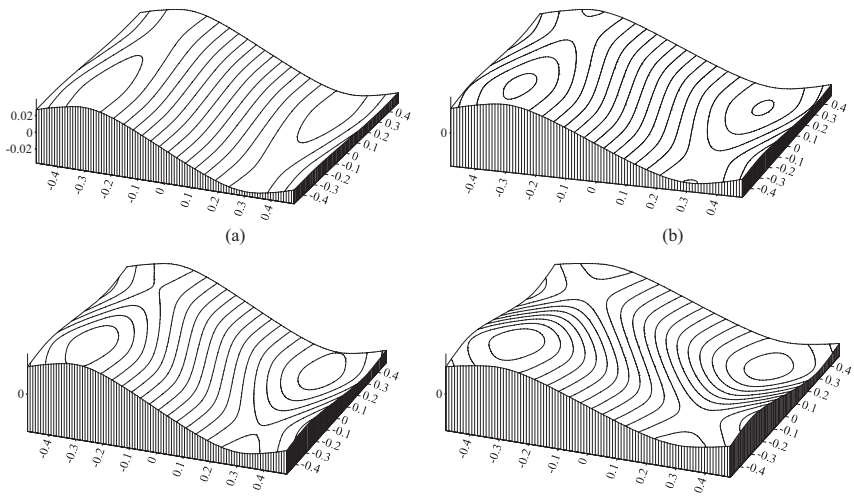

(c)
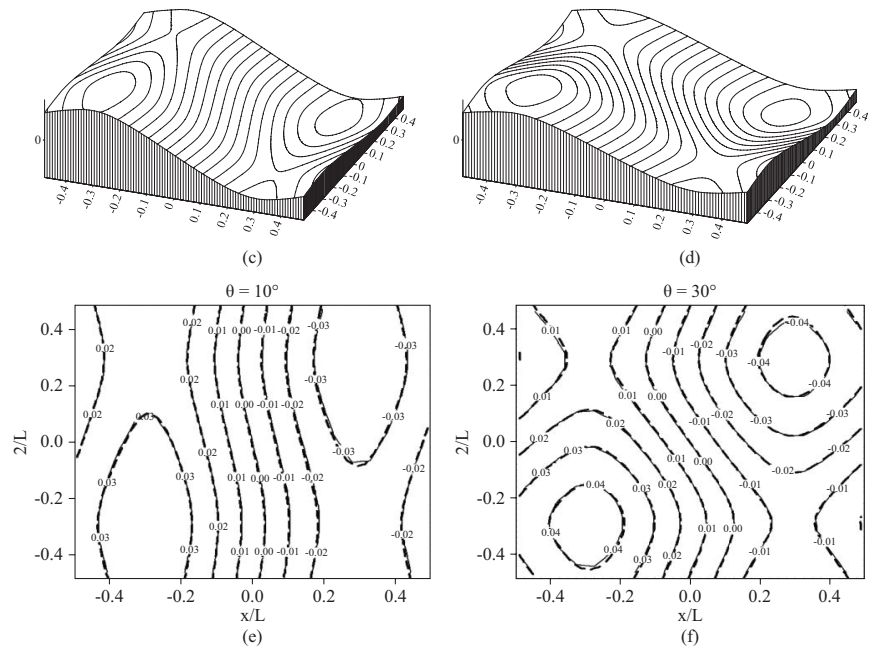

(d)

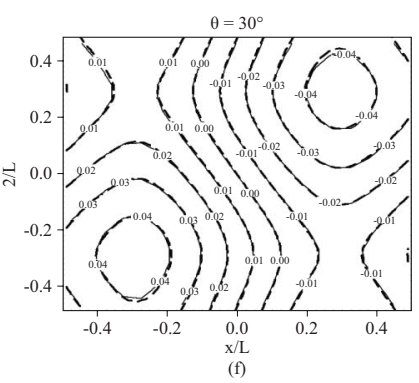

Fig. 11. The free surface contours of square-like waves with various excitation angles. The excitation frequency of tank is $1.5 \omega_{1}$. (a) $\theta=5^{\circ}$; (b) $\theta=10^{\circ}$; (c) $\theta=15^{\circ}$; (d) $\theta=30^{\circ}$; (e) $\theta=10^{\circ}$, solid line: $20 \times 10 \times 20$, dashed line: $40 \times 20 \times 40$; (f) $\theta=30^{\circ}$, solid line: $20 \times$ $10 \times 20$, dashed line: $40 \times 20 \times 40$.

further illustrate the free surface contours of square-like waves under different mesh numbers. These figures demonstrate the free surface profile of coarse meshes $(20 \times 10 \times 20$, solid line $)$ is almost the same as that of the finer meshes $(40 \times 20 \times 40$, dashed line). The coarse meshes, therefore, can be applied on the simulation of square-like waves.

Waves that slosh irregularly inside the tank are termed chaotic or irregular, waves. Irregular waves have been predicted when the excitation frequency lies within a tight band around the first natural frequency, although they were not observed in the experimental measurements of Faltinsen et al. [11]. In this study irregular waves could be found when the tank is excited by frequencies outside this limited range. Fig. 12 presents the results of a tank accelerated with an excitation angle of $\theta=10^{\circ}$ and an excitation frequency of $2.3 \omega_{1}$.

\section{Near Resonant Excitation}

Under a near resonant excitation, the waves will move along the tank walls in a clockwise or counterclockwise direction, they are referred to as "swirling" waves. The study of Faltinsen et al. [8] showed that the frequency domain for swirling waves right shifted away from the main resonance range with decreasing fluid depth, especially for $0.17<d_{0} / \mathrm{L}<$ 0.274. They also reported that the excitation frequencies for generating swirling waves are limited within a certain small range when the tank is excited by longitudinal, or diagonal,

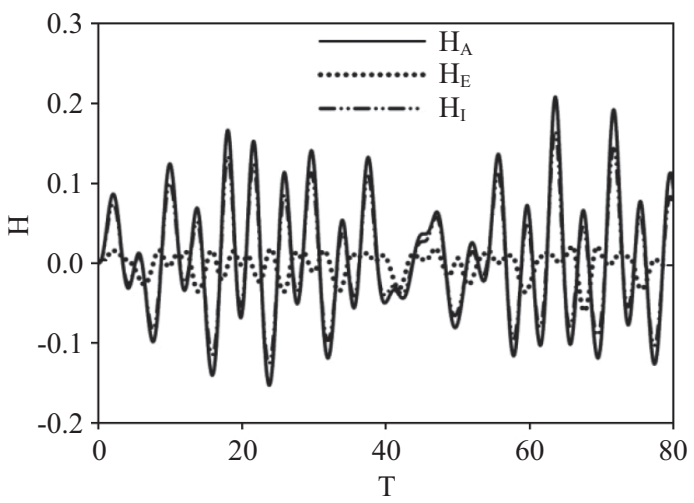

(a)

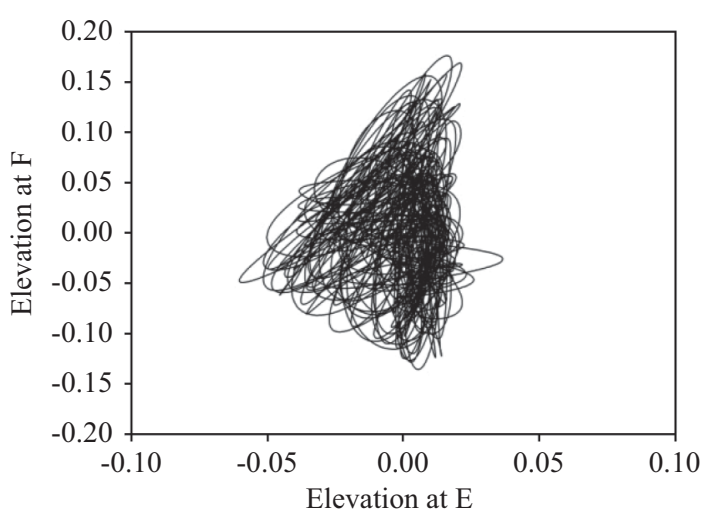

(b)

Fig. 12. The irregular waves. The tank is excited at $\theta=10^{\circ}, \omega=2.3 \omega_{1}$. (a) the wave elevation at point $A\left(H_{A}\right), E\left(H_{E}\right)$ and $I\left(H_{I}\right)$; (b) the pattern of irregular waves.

forcing. The existence of swirling waves for an excitation direction of $5^{\circ}$, and forcing frequency of $\omega / \omega_{1}=0.97$ is shown in Fig. 13. A beating phenomenon and a clear switching of direction can be seen in Figs. 13(a) and 13(b), respectively.

\section{Spectral Analysis}

In this section, a spectral analysis is performed in an attempt to identify the dominant frequencies for each type of sloshing wave. As the tank is excited under an excitation frequency close to the first natural frequency of the tank, the resultant phenomenon is called 'the primary resonance' of the tank. When the excitation frequency is moved away from the first natural frequency of the system, a secondary resonance might also be triggered due to a contribution of other natural modes of the system.

Fig. 14(a) presents the wave elevation and the corresponding spectral analyses of single-directional waves forced far from the first natural mode, $\omega=0.4 \omega_{1}$. The resulting motion is dominated by two frequencies, one near the primary resonance $\left(1.02 \omega_{1}\right)$ and the other near the excitation frequency $\left(0.42 \omega_{1}\right)$. Fig. 14(b) presents the results of square-like waves for a tank forced at $\omega=1.5 \omega_{1}$. Aside from the peaks at the primary resonance $\left(1.02 \omega_{1}\right)$ and excitation frequency $\left(1.53 \omega_{1}\right)$, two natural modes, $\omega_{3,0}$ and $\omega_{4,0}$ are also present, although 


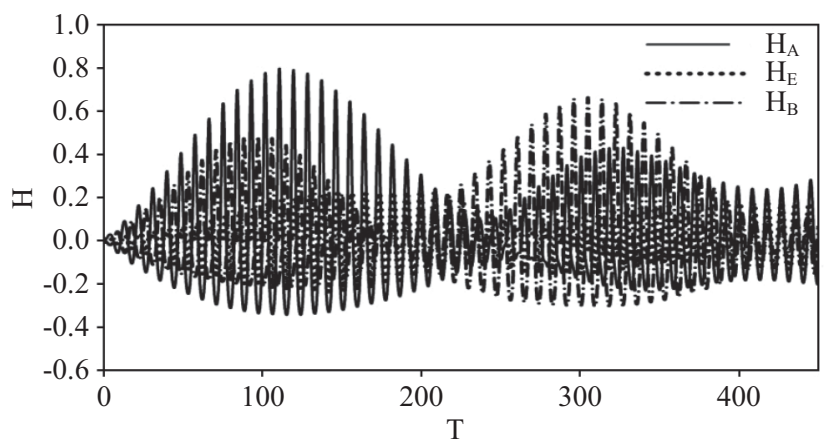

(a)

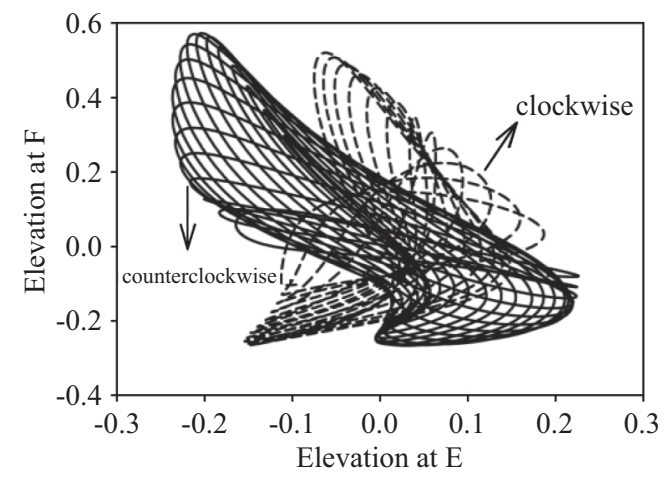

(b)

Fig. 13. The swirling waves. The tank is excited at $\theta=10^{\circ}, \omega=0.97 \omega_{1}$. (a) the wave history; (b) the pattern of swirling waves. Counterclockwise: $\mathrm{T}=\mathbf{4 0 \sim 2 0 0}$. Cloclwise: $\mathrm{T}=\mathbf{2 1 0} \mathbf{3 0 0}$.

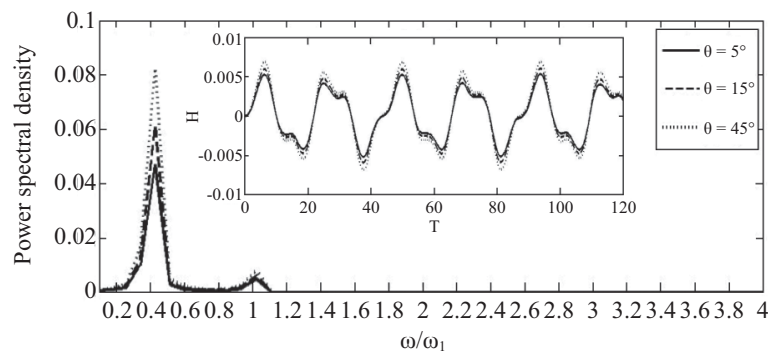

(a)

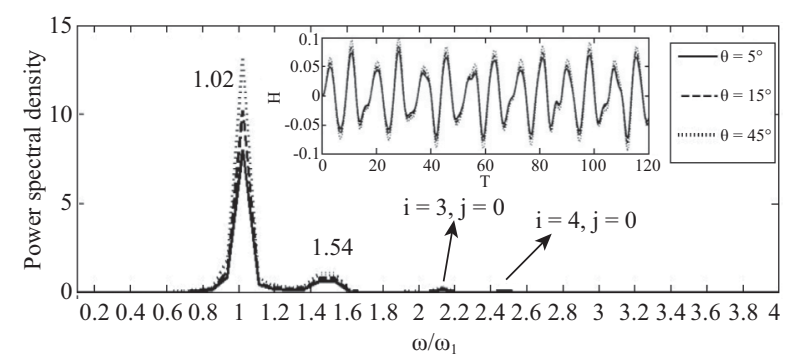

(b)

Fig. 14. Spectral analyses of wave elevation of corner $A$ for (a) diagonal waves and single-directional waves, $\omega=0.4 \omega_{1}$, and (b) squarelike waves, $\omega=1.5 \omega_{1}$. Subfigures: the wave history of each case.

they contain little energy. Thus, for diagonal, single direction and square-like waves, the major response frequencies are the first fundamental frequency and the excitation frequency.

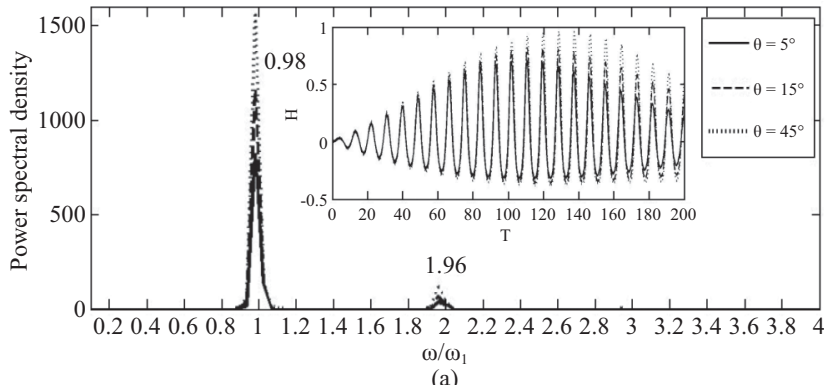

(a)

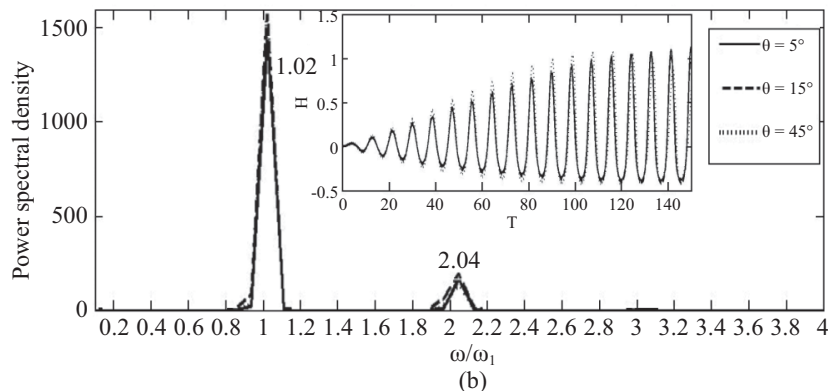

Fig. 15. Spectral analyses of wave elevation of corner A for swirling waves. (a) $\omega=0.97 \omega_{1}$ and (b) $\omega=1.04 \omega_{1}$. Subfigures: the wave history of each case.

Figs. 15(a) and 15(b) show the spectral analysis for swirling waves in a tank forced at $\omega=0.97 \omega_{1}$ and $\omega=1.04 \omega_{1}$, respectively. The figures clearly show resonance peaks corresponding to the primary natural frequency, and also $2 \omega_{1,0}$ which is slightly smaller than the natural frequency $\omega_{2,2}$. The spectral analyses of swirling waves consistently show the occurrence of a resonance peak corresponding to $\omega_{2,2}$, and this secondary resonance may be related to the development of the swirling wave in the tank. This finding will be validated with more simulation examples presented in later sections.

Fig. 16(a) depicts the spectral analysis of irregular waves sloshing in a tank accelerated by an excitation frequency of 1.8 $\omega_{1,0}$. In addition to the primary resonance and a peak corresponding to the excitation frequency, there is another resonant peak attributed to the odd natural mode $\omega_{3,0}$. Fig. 16(b) plots the spectral analysis for a tank sloshing with an excitation angle of $45^{\circ}$, and an excitation frequency of $2.3 \omega_{1}$, and in this case not only is the resonant peak $\omega_{3,0}$ observed, but a peak corresponding to $\omega_{5,0}$ is also present. It appears to be common for the occurrence of irregular waves to be related to the appearance of odd natural mode responses (Wu and Chen, [26]).

\section{The Limitation of the Present Numerical Model and the Requirement of CPU Time}

The simulations for cases with strong shallow-fluid sloshing and large excitation amplitude were failed to satisfactorily converge and thus these simulations are beyond the current capability of our model. Fig. 17 presents the results of a tank under shallow water depth $\left(d_{0} / \mathrm{L}=0.1\right)$. For a non-resonant oscillation, the proposed numerical scheme can be applied with $\mathrm{A} / d_{0}=2.2$ (Fig. 17(a)). However, the limit of $\mathrm{A} / d_{0}$ 


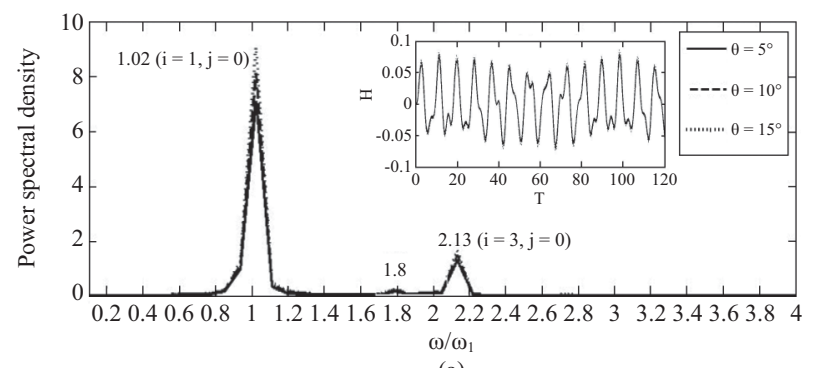

(a)

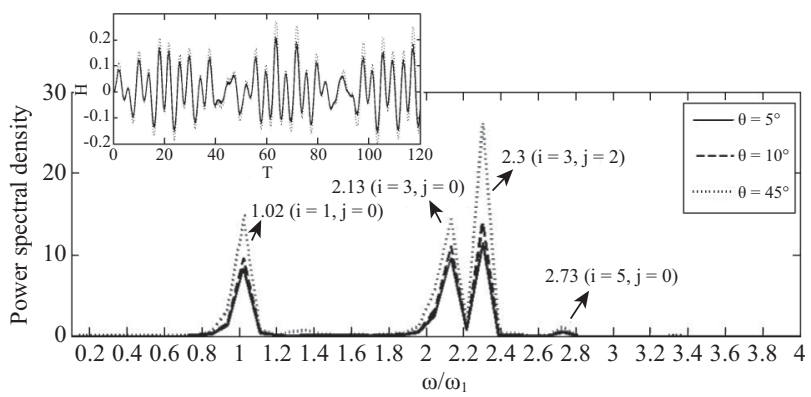

(b)

Fig. 16. Spectral analyses of wave elevation of corner A for irregular waves. (a) $\omega=1.8 \omega_{1}$ and (b) $\omega=2.3 \omega_{1}$. Subfigures: the wave history of each case.

becomes 0.05 in a near-resonant oscillation due to large nonlinear effect appeared in the shallow water depth, as shown in Fig. 17(b). Figs. 17(c) and (d) further reveal the angle of wave slopes $\left(\theta_{w}\right)$ of the free surface along the south wall and diagonal line of the tank for the resonant sloshing with $\mathrm{A} / d_{0}=$ 0.1 , respectively. The angle of wave slope $\left(\theta_{w}\right)$ is defined as the angle between the free surface and the horizontal plane. The excitation angle is $5^{\circ}$ and thus, the maximum wave slope occurs along the south (north) wall of the tank and the limitation of the wave slope of this work is about $40^{\circ}$.

The requirements of CPU time of each type of sloshing waves are listed in Table 1. The AMD Phenom II X4 955 is used for computing under Linux Operating system. Table 1 shows the swirling waves need more CPU time than the others and the reason is trivial that more iterations are required when a tank is excited under near resonant frequencies.

\section{CONCLUSIONS}

The following conclusions have been reached:

1. An efficient numerical scheme with high accuracy has been developed and the method can be used to study a 3D tank excited by complete 3D motions with simultaneous action of six-degree-of-freedom.

2. Benchmark tests with previous studies indicate that the current numerical scheme has acceptable levels of accuracy.

3. The results of Faltinsen et al. $[8,10]$ indicate that squarelike and irregular waves will be present when the tank is under near resonant excitation conditions. However, in the
Table 1. The requirement of CPU time for different sloshing waves simulated up to 60 seconds, mesh numbers: $20 \times 10 \times 20, \theta=10^{\circ}, d_{0} / \mathrm{L}=0.25$.

\begin{tabular}{|c|c|c|}
\hline Wave type & Time step & CPU time (hours) \\
\hline Single-directional waves & 0.006 & 9.17 \\
\hline Square-like waves & 0.006 & 8.66 \\
\hline Swirling waves & 0.003 & 19.84 \\
\hline Irregular waves & 0.003 & 17.62 \\
\hline
\end{tabular}

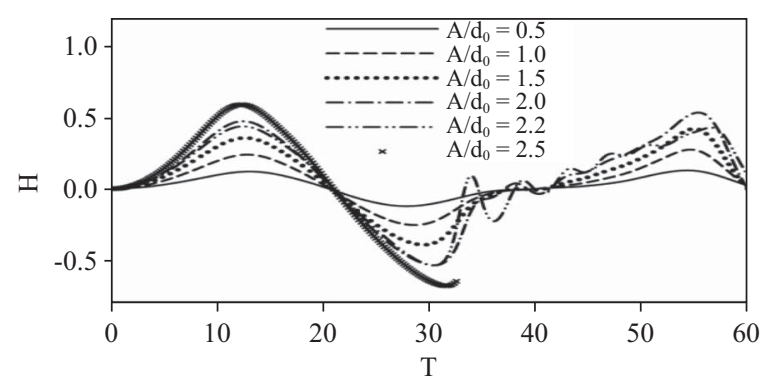

(a)

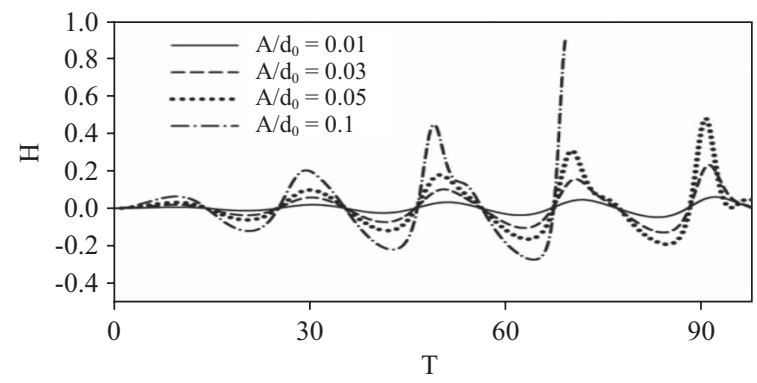

(b)

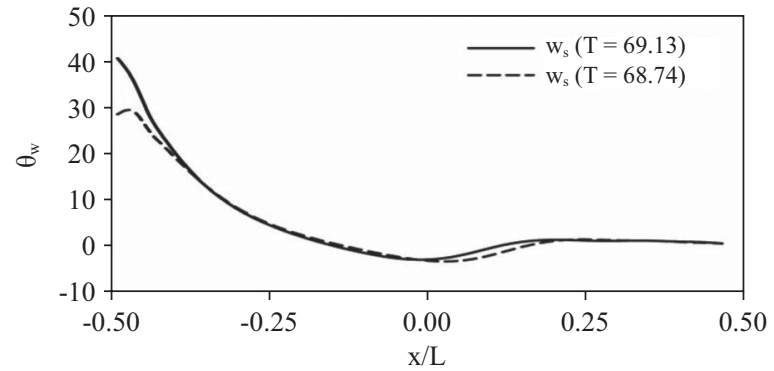

(c)

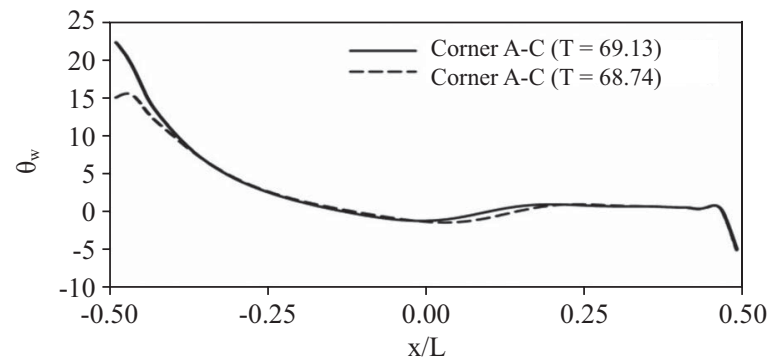

(d)

Fig. 17. The limitation of the present numerical scheme under shallow water depth $\left(d_{0} / L=0.1\right)$. The wave history at point $A$. (a) $\omega_{x}=$ $\omega_{z}=0.5 \omega_{1}$. (b) $\omega_{x}=\omega_{z}=0.97 \omega_{1}$. The angle of wave slope $\left(\theta_{w}\right)$ of $\mathrm{A} / d_{0}=0.1$ (c) along south wall (Ws) and (d) diagonal line of the tank. Excitation angle $\theta=5^{\circ}$. 
present study, in tanks suffering horizontal ground motion at various excitation angles, square-like and irregular waves not only occur near resonant frequency excitation, but also at excitation frequencies far away from the first natural frequency of the system.

4. The difference between diagonal waves and singledirectional waves is due to the tank being excited at various excitation angles.

5. The free surface contours of square-like waves show different terraced planes under various excitation angles, and the terraced planes are most obvious when $\theta=30^{\circ}$ and $\theta=$ $45^{\circ}$.

6. Swirling waves occur when the tank is under a near resonant excitation frequency, except when the excitation angle is close to $45^{\circ}$. In this case, the phenomenon is absent. The swirling waves will change swirling directions (counterclockwise to clockwise) according to the phase lag between the wave motion and the motion of the tank.

7. The spectral analyses for different sloshing waves have shown that (a) only the primary resonance and excitation frequencies influence the sloshing system of diagonal, single-directional and square-like waves; (b) for the sloshing system of swirling waves, not only the primary mode, but the secondary mode also appears in the spectral diagram and causes a secondary resonance; (c) in the spectral analysis of irregular waves, the odd natural modes $(i=1,3$, $5 \ldots, \mathrm{j}=0)$ are generated and have a significant influence on the sloshing system.

\section{REFERENCES}

1. Abramson, H. N., "The dynamics of liquids in moving containers," NASA Rep. SP 106 (1966).

2. Aliabadi, S., Johnson, A., and Abedi, J., "Comparison of finite element and pendulum modelsfor simulation of sloshing," Computers Fluids, Vol. 23, pp. 535-545 (2003)

3. Cariou, A. and Casella, G., "Liquid sloshing in ship tanks: a comparative study of numerical simulation," Marine Structures, Vol. 12, pp. 183-189 (1999).

4. Celebi, M. S. and Akyildiz, H., "Nonlinear modeling of liquid sloshing in moving rectangular tank," Ocean Engineering, Vol. 29, pp. 1527-1553 (2002).

5. Chen, B. F. and Chiang, S. W., "Complete 2D and fully nonlinear analysis of sloshing fluid in a rigid tank," Journal of Engineering Mechanics, Vol. 125, No. 1, pp. 70-78 (1999).

6. Chen, B. F. and Nokes, R., "Time-independent finite difference analysis of fully non-linear and viscous fluid sloshing in a rectangular tank," Journal of Computational Physics, Vol. 209, pp. 47-81 (2005).

7. Faltinsen, O. M., "A nonlinear theory of sloshing in rectangular tanks," Journal of Ship Research, Vol. 18, pp. 224-241 (1974).

8. Faltinsen, O. M., Rognrbakke, O. F., and Timokha, A. N., "Resonance three-dimensional nonlinear sloshing in a square-base basin," Journal of
Fluid Mechanics, Vol. 487, pp. 1-42 (2003).

9. Faltinsen, O. M., Rognrbakke, O. F., and Timokha, A. N., "Classification of three-dimensional nonlinear sloshing in a square-base tank with finite depth," Journal of Fluids and Structures, Vol. 20, pp. 81-103 (2005a).

10. Faltinsen, O. M., Rognrbakke, O. F., and Timokha, A. N., "Resonant three-dimensional nonlinear sloshing in a square-base basin. Part 2. Effect of higher modes," Journal of Fluid Mechanics, Vol. 523, pp. 199-218 (2005b).

11. Faltinsen, O. M., Rognrbakke, O. F., and Timokha, A. N., "Resonant three-dimensional nonlinear sloshing in a square-base basin. Part 3. Base ratio perturbations," Journal of Fluid Mechanics, Vol. 551, pp. 93-116 (2006).

12. Feng, G. C., "Dynamic loads due to moving liquid," American Institute of Aeronautics and Astronautics, Paper No. 73-409 (1976).

13. Frandsen, J. B., "Sloshing motions in excited tanks," Journal of Computational Physics, Vol. 196, pp. 53-87 (2004).

14. Hirt, C. W. and Nichols, B. D., "Volume of fluid method for the dynamics of free boundaries," Journal of Computational Physics, Vol. 39, pp. 201225 (1981).

15. Hirt, C. W., Nicholas, B. D., and Romero, N. C., "SOLA-a numerical solution algorithm for transient fluid flows," Los Alamos Scientific Laboratory, Report LA-5852 (1975).

16. Hung, T. K. and Chen, B. F., "Nonlinear hydrodynamic pressure on dams during earthquake," Journal of Engineering Mechanics, ASCE, Vol. 116, No. 6, pp. 1372-1391 (1990).

17. Hung, T. K. and Wang, M. H., "Nonlinear hydrodynamic pressure on rigid dams motion," Journal of Engineering Mechanics, ASCE, Vol. 113, No. 4, pp. 482-499 (1987).

18. Ibrahim, R. A., Pilipchuk, V. N., and Ikeda, T., "Recent advances in liquid sloshing dynamics," Applied Mechanics Research, Vol. 54, No. 2, pp. 133-199 (2001).

19. ISSC 1997 Report of Committee I.2 "Loads," in: Moan, T. and Berge, S. (Eds.), Proceeding 13th Intl Ship and Offshore Structures Congress, Pergamon, Vol. 1, pp. 59-122 (1997).

20. Kim, Y., "Numerical simulation of sloshing flows with impact loads," Applied Ocean Research, Vol. 23, pp. 53-62 (2001).

21. Miles, J. W., "Faraday waves: rolls versus squares," Journal of Fluid Mechanics, Vol. 269, pp. 353-371 (1994).

22. Nakayama, T. and Washizu, K., "Nonlinear analysis of liquid motion in a container subjected to forced pitching oscillation," International Journal for Numerical Methods in Engineering, Vol. 15, pp. 1207-1220 (1980).

23. Ockendon, J. R., Ockendon, H., and Waterhouse, D. D., "Multi-mode resonances in fluids," Journal of Fluid Mechanics, Vol. 315, pp. 317-344 (1996).

24. Turnbull, M. S., Borthwick, A. G. L., and Eatock Taylor, R., "Numerical wave tank based on a $\sigma$-transformation finite element inviscid flow solver," International Journal of Numerical Method in Fluid, Vol. 42, pp. 641-663 (2003).

25. Van Doormaal, J. R. and Raithby, G. D., "Enhancements of the SIMPLE method for predicting incompressible fluid flows," Numerical Heat Transfer, Vol. 7, pp. 147-163 (1984).

26. Wu, C. H. and Chen, B. F., "Sloshing waves and resonance modes of fluid in a 3D tank by a time-independent finite difference method," Ocean Engineering, Vol. 36, pp. 500-510 (2009).

27. Wu, G. X., Ma, Q. W., and Taylor, R. E., "Numerical simulation of sloshing waves in a 3D tank based on a finite element methods," Applied Ocean Research, Vol. 20, pp. 337-355 (1998). 